\title{
Using neutrons to measure keV temperatures in highly compressed plastic at multi-Gbar pressures
}

J. Nilsen ${ }^{1}$, B. Bachmann ${ }^{1}$, G. B. Zimmerman ${ }^{1}$, R. Hatarik ${ }^{1}$, T. Döppner ${ }^{1}$, D. Swift ${ }^{1}$, J. Hawreliak ${ }^{2}$, G. W. Collins $^{1}$, R. W. Falcone ${ }^{3}$, S. H. Glenzer ${ }^{4}$, D. Kraus ${ }^{3}$, O. L. Landen ${ }^{1}$, and A. L. Kritcher ${ }^{1}$

${ }^{1}$ Lawrence Livermore National Laboratory, Livermore, CA 94551

${ }^{2}$ Washington State University, Pullman, WA 99164

${ }^{3}$ University of California Berkeley, Berkeley, CA 94720

${ }^{4}$ Stanford Linear Accelerator Center, Menlo Park, CA 94305

\begin{abstract}
We have designed an experiment for the National Ignition Facility to measure the Hugoniot of materials such as plastic at extreme pressures. The design employs a strong spherically converging shock launched through a solid ball of material using a hohlraum radiation drive. The shock front conditions can be characterized using X-ray radiography until background from shock coalescence overtakes the backlit signal. Shock coalescence at the center is predicted to reach tens of Gbars and can be further characterized by measuring the X-ray self-emission and 2.45 $\mathrm{MeV}$ neutrons emitted from the shock flash region. In this simulation design work the standard plastic sphere is replaced with a deuterated polyethylene sphere, $\mathrm{CD}_{2}$, that reaches sufficiently high densities and temperatures in the central hot spot to produce neutrons from Deuterium-Deuterium (DD) fusion reactions that can be measured by a neutron time of flight spectrometer (nTOF) and act as a temperature diagnostic. This paper focuses on the design of these experiments, based on an extensive suite of radiation-hydrodynamics simulations, and the interpretation of the predicted DD neutron signals. The simulations predict mean temperatures of $1 \mathrm{keV}$ in the central hot spot with mean densities of $33 \mathrm{~g} / \mathrm{cc}$ and mean pressures of $25 \mathrm{Gbar}$. A preliminary comparison with early experimental results looks promising with an average ion temperature of $1.06 \pm 0.15 \mathrm{keV}$ in the central hot spot estimated from the nTOF spectral width and measured neutron yield of $7.0( \pm 0.5) \times 10^{9}$ DD neutrons.
\end{abstract}

\section{Introduction}

In gas-giant planets, brown dwarfs, and highly evolved stars material can reach extreme pressures that exceed 100 Mbar and reach well into the Gbar regime [1-3]. Laboratory measurements of matter at high energy density is of great importance in understanding the structure and evolution of these objects and for accurate modeling of matter at extreme conditions.

At Lawrence Livermore National Laboratory (LLNL) the Gbar experimental platform [4] has been developed and demonstrated to study the shock compression (Hugoniot) of material compressed to near Gbar pressures in a spherically converging geometry using streaked X-ray radiography and X-ray Thomson scattering to measure the material parameters. In these experiments, the NIF laser heats the inside of a gold hohlraum and creates an X-ray radiation drive which ablates an outer ablator and sends a strong spherical shock into a solid sphere of material. The shock front conditions are characterized using X-ray radiography to determine the pressure and density along the shock Hugoniot as the shock travels to the center of the solid 
sphere. As the shock travels toward the center the pressure increases like $\sim 1 / \mathrm{r}$ and the temperature at the shock front increases, resulting in X-ray self-emission, which ultimately overwhelms the signal from X-ray radiography.

When the shock coalesces at the near center of the target, mean pressures and temperatures of 25 Gbars and $1 \mathrm{keV}$ are reached. These conditions at shock coalescence are further characterized by measuring the X-ray self-emission and neutrons (for $\mathrm{CD}_{2}$ targets) emitted from the high temperature hot-spot plasma. In this work we describe a detailed design based on radiationhydrodynamics simulations, using a solid deuterated polyethylene target, $\mathrm{CD}_{2}$, to create 2.45 $\mathrm{MeV}$ neutrons from deuterium-deuterium fusion reactions. These neutrons are Doppler broadened, and can be measured via neutron time-of-flight detectors to give a burn-averaged ion temperature in the central hot spot, where the pressures are predicted to exceed $10 \mathrm{Gbar}$. We describe below the results of radiation-hydrodynamics simulations, which agree very well with preliminary experimental results that predict mean temperatures of $1 \mathrm{keV}$ in the central hot spot with mean densities of $33 \mathrm{~g} / \mathrm{cc}$ and mean pressures of 25 Gbar. These combined results are an important step toward understanding the plasma conditions in the central hot spot in the laboratory and the high- $(\rho, \mathrm{T})$ conditions of planetary and stellar interiors.

\section{Radiation hydrodynamic simulations}

In this section we present radiation hydrodynamics design simulations using the Lasnex code [5] which motivate the new Gbar capability, shown in Fig. 1, and give the hot-spot plasma conditions and dynamics for this platform. These simulations are capsule-only simulations that use a frequency dependent radiation drive that surrounds the capsule. This drive is derived from integrated Hohlraum simulations using a Detailed Configuration Accounting (DCA) atomic physics model [6]. This radiation drive was modified to account for the fact that 16 of the 192 drive beams are used to drive the back-lighter for the streaked radiography. The NIF laser drive uses 1.19 MJ of energy in a two-step pulse described in Ref. 7 to produce the X-ray drive in the hohlraum. The radiation temperature as a function of time is plotted in Fig. 2. The peak radiation temperature is predicted to be $297 \mathrm{eV}$ at a time of 5.03-ns. The radiation drive launches two shocks into the ablator, which coalesce inside of the solid plastic ball into a single strong shock. To model this in one dimension (1D) the simulations use the LEOS 5358 tabular equation-ofstate (EOS) table for the doped GDP ablator and LEOS 5100 for the $\mathrm{CD}_{2}$ sphere. More detailed 
discussion about the sensitivity of the calculations to the use of different EOS tables can be found in Ref. 4 but the radial dependence of the pressure is only weakly dependent on the EOS used. The radiation transport is modeled using multi-group, discrete-ordinates ( $\mathrm{Sn}$ ) radiation transport [8], with 100 radiation groups. The neutron production is calculated using the known $\sigma \mathrm{v}$ reactivities (product of cross section and ion velocity) together with the calculated temperatures and densities from the hydrodynamic simulations. There is no significant energy produced by the neutron production and it does not affect the hydrodynamics.

The simulations predict that the initial shock of 150 Mbar launched by the ablation pressure reaches the outer radius $(855 \mu \mathrm{m})$ of the $\mathrm{CD}_{2}$ sphere at $4.2 \mathrm{~ns}$ and continues to travel toward the center, reaching a radius of $100 \mu \mathrm{m}$ at $8.35 \mathrm{~ns}$ with a pressure of $1.0 \mathrm{Gbar}$. The shock reaches the center 170 ps later at $8.520 \mathrm{~ns}$ creating the central hot spot. This time agrees very well with the measured X-ray bang time of $8.520 \mathrm{~ns}(+30 \mathrm{ps},-10 \mathrm{ps})$ for the hot spot measured by the Streaked Polar Instrumentation for Diagnosing Energetic Radiation (SPIDER) diagnostic [9]. The simulations predict that the peak of the neutron production occurs 8 psec later at $8.528 \mathrm{~ns}$. In this paper we use the neutron time-of-flight (TOF) spectra as a temperature diagnostic for the central hot spot and simulations to predict the plasma conditions in the hot spot. This works concentrates on the time period from 20 psec before the shock hits the center to about 100 psec afterwards. In the remaining figures we define the zero of time as the time of peak neutron production.

To appreciate the relative time scales Figure 3 shows the calculated neutron production rate versus time. One notices that the predicted rise time is only $6.2 \mathrm{psec}$ for $10-90 \%$ production while the full-width half-maximum (FWHM) is 75 psec because there is a slower falling tail. To understand the neutron production and the corresponding plasma conditions occurring in the hot spot Figures 4 - 7 plot the predicted pressure, neutron production rate, ion temperature, and density versus radius every 20 psec starting at -20 psec to +100 psec relative to the time of peak neutron production. We should point out that the electron and ion temperatures are very similar through out the $\mathrm{CD}_{2}$ material.

Figure 4 shows snapshots of the predicted pressure versus radius at different times. At $t=-20$ psec the peak pressure of the incoming shock is 6.0 Gbar at a radius of $12 \mu \mathrm{m}$ while at $\mathrm{t}=0$ the peak pressure of the outgoing shock has jumped to $68 \mathrm{Gbar}$ at a radius of $5.3 \mu \mathrm{m}$. The outgoing shock maintains pressures near 40 Gbar over the next 60 psec and then starts to decay. By $\mathrm{t}=$ $160 \mathrm{psec}$ the peak pressure is still 17 Gbar over quite a large region extending out to a radius of more than $40 \mu \mathrm{m}$. 
Fig. 5 shows the neutron production rate in units of neutrons per psec per $\mu \mathrm{m}$ versus radius. At $\mathrm{t}=-20$ psec the rate is very low $\left[\cong 10^{5} /(\mathrm{ps}-\mu \mathrm{m})\right]$ but quickly increases by two orders to a peak rate of $2 \times 10^{7} /(\mathrm{ps}-\mu \mathrm{m})$ at $\mathrm{t}=0$ and a radius of $5 \mu \mathrm{m}$. One can see that the peak rate falls but spreads out spatially as the time increases.

Since the neutron reaction rate depends strongly on the ion temperature, Fig. 6 plots the predicted ion temperature $T_{i}$ versus radius. The ion temperature in the central hot spot quickly rises from $0.55 \mathrm{keV}$ right before the shock hits the center to $3 \mathrm{keV}$ at $\mathrm{t}=0$ but quickly falls off to 2 and $1.5 \mathrm{keV}$ in the next 40 psec. The ion temperature does persist above $0.7 \mathrm{keV}$ for 100 psec out to a radius of $50 \mu \mathrm{m}$. The fast rise and fall of the temperature accounts for the fast rise time and initial falloff of the neutron production rate in Fig. 3. The long tail in the neutron production is consistent with a large spatial region with temperatures near $0.7 \mathrm{keV}$.

Figure 7 plots the density versus radius. At $\mathrm{t}=-20 \mathrm{ps}$ one observes the unshocked $\mathrm{CD}_{2}$ at density $1.05 \mathrm{~g} / \mathrm{cc}$ for radii less than $10 \mu \mathrm{m}$. For larger radii the shocked material has density near $10 \mathrm{~g} / \mathrm{cc}$. After the incoming shock reaches the center the density jumps to $30 \mathrm{~g} / \mathrm{cc}$ and the density continues to increase during the outgoing shock with densities reaching $55 \mathrm{~g} / \mathrm{cc}$ at $\mathrm{t}=80 \mathrm{psec}$ with large regions having densities above $40 \mathrm{~g} / \mathrm{cc}$ for times out to $160 \mathrm{psec}$. Unlike the ion temperature that falls off very quickly after the shock reaches the center the density climbs and persists for a long time because the material is re-shocked due to the incoming shock. Since the neutrons production rate is proportional to the square of the density this helps explain the slower falloff in the neutron production rate.

\section{Experimental platform and nTOF results}

Motivated by the design simulations, experiments were developed to look at these unique high-temperature and density conditions. A detailed description of the experimental setup can be found in Ref. 4. A schematic of the experimental configuration is shown in Fig. 1a. In these experiments, solid targets are compressed and heated in an indirect drive laser geometry using 172 laser beams incident on an Au hohlraum. In the first experiments [4] the targets consisted of solid $\mathrm{CH}$ spheres $965 \mu \mathrm{m}$ in radius covered by a $185 \mu \mathrm{m}$ thick $\mathrm{CH}$ ablator with a graded Ge dopant. In the present experiments the $\mathrm{CH}$ sphere is replaced with a deuterated polyethylene sphere, $\mathrm{CD}_{2}$, of radius $855-\mu \mathrm{m}$. The $\mathrm{CD}_{2}$ sphere was made by compressing $\mathrm{CD}_{2}$ powder into a cylinder of solid density that was then machined into a spherical target. The $\mathrm{CD}_{2}$ sphere was 
coated with a $285-\mu \mathrm{m}$ thick graded Ge doped $\mathrm{CH}$, or glow-discharge polymer (GDP), ablator coated onto the spheres similar to the National Ignition Campaign (NIC) ablators. The coating of the $\mathrm{CD}_{2}$ spheres was performed at General Atomics (GA). The Ge dopant is used to mitigate $\mathrm{X}$ ray preheat induced by Au M-shell emission from the gold hohlraum.

The experiments use a standard NIC scale $5.75 \mathrm{~mm}$ hohlraum (diameter $=5.75 \mathrm{~mm}$ and height $=9.42 \mathrm{~mm}$ ) with a $0.03 \mathrm{mg} / \mathrm{cc}{ }^{4} \mathrm{He}$ gas fill and $3.375 \mathrm{~mm}$ diameter laser entrance hole. The hohlraum is driven by 172, 351-nm laser beams with a two shock pulse shape that can be described as an 1-ns duration, 75-TW initial square pulse that then ramps up to 330 TW over 2ns and maintains $330 \mathrm{TW}$ over the next 2-ns [7]. The total drive energy and power incident on the hohlraum walls is about $\cong \square 1.19 \mathrm{MJ}$ and $330 \mathrm{TW}$, respectively. These drive conditions result in a predicted peak hohlraum radiation temperature of about $300 \mathrm{eV}$, see Fig. 2. This pulse is based on a similar two shock pulse shape that uses the first pulse to ablate enough $\mathrm{CH}$ from the sphere to prevent early wall motion from imprinting on the sphere for the low gas fill used in the hohlraum [10]. The two shocks coalesce in the Ge doped $\mathrm{CH}$ ablator so that the $\mathrm{CD}_{2}$ sphere see a single strong shock.

Figure $1 \mathrm{~b}$ shows a simulated streaked radiograph across a diameter of the sphere from Ref. 4 with the hot spot formed as the shock reaches the center. In the radiograph one can see the shock wave converging toward the center of the capsule with the bright vertical white line showing the X-ray emission from the hot spot. When the shock reaches the center of the $\mathrm{CD}_{2}$ target the temperatures and densities are high enough to produce neutrons. The $2.45 \mathrm{MeV}$ neutrons from the deuterium-deuterium (DD) reactions are measured using three neutron time of flight (nTOF) diagnostics that are located 18 to 22 meters from the center of the hohlraum. The best statistics are from the nTOF [11] at the south pole that is 17.976 meters from target. The temporal width of the nTOF spectrum is used to determine an average ion temperature for the hot spot region that produces the neutrons $[12,13]$.

Figure 8 shows the measured nTOF spectrum at 17.976 meters from the target. The measured spectrum is shown by the solid curve and is normalized to an intensity of 1 . From the spectral width the estimated ion temperature is $1.06 \pm 0.15 \mathrm{keV}$ with a measured neutron yield of 7.0 $( \pm 0.5) \times 10^{9} \mathrm{DD}$ neutrons. The simulated spectrum including the effect of the instrument response function (IRF) is shown as a black dashed curve, and is in good agreement with the experimental data, also normalized to an intensity of 1 . The ion temperature can be estimated from the width of the nTOF spectrum with the formula $T_{i o n}=[1.285 \mathrm{x} \Delta t / D]^{2}$ where $T_{\text {ion }}$ is the 
ion temperature in $\mathrm{keV}, \Delta \mathrm{t}$ is the full-width at half maximum (FWHM) for the temporal width in nsec, and D is the distance of the target to the detector in meters assuming there is no spreading due to the IRF [13]. As will be discussed later the mean ion temperature in the simulation is 1.07 $\mathrm{keV}$. The simulations predict a total neutron production of $1.25 \times 10^{10} \mathrm{DD}$ neutrons, which is about a factor of 2 higher than the measured value, which is very good agreement given the highly non-linear dependence of the neutron output on the ion temperature.

\section{Using distribution functions to understand mean conditions in the central hot spot}

Given the large gradients in time and space we created distribution functions versus pressure, ion temperature, density, and radius for the neutron production to help understand the plasma conditions when the neutrons are produced in the central hot spot. To create the distribution functions we looked at how many neutrons were created in every zone in space at every time step and tallied that information to fill the distribution function. The distribution functions were created so that the integral over the dependent variables would yield the total number of 2.45 $\mathrm{MeV}$ DD neutrons predicted. Figure 9 shows the distribution function, $\mathrm{dN}(2.45 \mathrm{MeV}) / \mathrm{dP}$, in units of neutrons $\mathrm{N}$ per Gbar versus pressure $\mathrm{P}$ in Gbars. A peak in this distribution function gives the hot spot pressure at which the maximum production of $2.45 \mathrm{MeV}$ neutrons is occurring. The black dashed curve shows the total distribution function over the entire simulated volume and time interval out to $11 \mathrm{~ns}$ on a scale where peak neutron production is at a time of $8.520 \mathrm{~ns}$. One striking feature is the two peaks, one at 7.75 Gbar and the second peak at 37.2 Gbar. To understand this we also created a distribution function for a simulation that ends 40 psec after the peak of the neutron production rate $(\mathrm{t}=0 \mathrm{ps}$ in Fig. 3), as shown by the red solid curve in Fig. 9. According to the simulations, $28 \%$ of the total neutrons have been made by this time. The high pressure peak corresponds to the neutrons produced in the initial creation of the hot spot when the shock reaches the center and starts to rebound, while the lower pressure peak is from the slower decaying tail of the neutron production shown in Fig. 3. This slower component consists of the neutrons produced from the outgoing shock that maintains a high density even as the temperature starts to fall quickly. In the subsequent Figures we will look at the total neutron production and compare it with the fast component ( $\mathrm{t}<40$ ps after peak neutron production). Using the neutron distribution function as a weighting factor, a mean pressure can be calculated by multiplying the pressure by the distribution function, integrating over pressure, and dividing 
by the total number of neutrons created. This gives a mean pressure of 24.6 Gbar for neutron production in the plasma. If we now calculate a mean pressure during the fast component $(\mathrm{t}<40$ psec) the mean pressure is 40.8 Gbar while the mean pressure during the slow component $(\mathrm{t}>40$ psec) is 16.4 Gbar.

For the ion temperature Fig. 10 shows the neutron distribution, $\mathrm{dN}_{\mathrm{d}} \mathrm{dT}_{\mathrm{i}}$, in neutrons per $\mathrm{keV}$ versus ion temperature $\mathrm{T}_{\mathrm{i}}$ in $\mathrm{keV}$ for the total distribution (dashed) and the fast component (solid). A peak in this distribution function gives the hot spot temperature at which the maximum production of $2.45 \mathrm{MeV}$ neutrons is occurring. The total distribution peaks at an ion temperature of $620 \mathrm{eV}$. However the mean temperature, weighted by the neutron production rate distribution function, gives an ion temperature of $1.07 \mathrm{keV}$ in good agreement with the temperature of 1.06 keV measured by the nTOF. In Fig. 8 the dashed curve shows the simulated nTOF spectrum convoluted with the instrument response function. This agrees well with the measured spectrum (solid curve) and the mean temperatures are very consistent.

To better understand the value of $\mathrm{dN}(2.45 \mathrm{MeV}) / \mathrm{dT}_{\mathrm{i}}$ during the initial hot spot the solid curve in Fig. 10 shows the neutron production versus ion temperature $\mathrm{T}_{\mathrm{i}}$ for $\mathrm{t}<40$ ps. For the fast component the temperature rises sharply at $1.2 \mathrm{keV}$ and most of the neutron production is above this ion temperature $T_{i}$ with a mean value of $2.03 \mathrm{keV}$. Looking at the neutrons produced after 40 ps the mean ion temperature is $0.688 \mathrm{keV}$. Looking at the nTOF spectrum in Fig. 8, we split the simulated spectrum into a fast component for neutrons produced for a simulation that ends 40 psec after the peak of the neutron production and a slow component for all the neutrons produced after 40 psec. The fast component contributes to broadening the wings of the spectrum while the slow component has a narrower width consistent with the lower temperature. With sufficient resolution in the nTOF spectrum one can fit the nTOF spectrum with a two temperature fit and use the width in the wings of the distribution to determine the fast component of the hot spot temperature.

For the density Fig. 11 plots the neutron distribution, dN/d $\rho$, in neutrons per g/cc versus density $\rho$ in $\mathrm{g} / \mathrm{cc}$. The dashed curve shows the total distribution with most of the density between 18 and $50 \mathrm{~g} / \mathrm{cc}$ and a mean density of $33.5 \mathrm{~g} / \mathrm{cc}$. The fast component (solid curve) has a similar range of densities and a mean density of $31.8 \mathrm{~g} / \mathrm{c}$. This is consistent with the density staying high for a long period of time.

For the spatial distribution Fig. 12 plots the neutron distribution, dN/dR, in neutrons per $\mu \mathrm{m}$ versus radius $\mathrm{R}$ in $\mu \mathrm{m}$. For the total distribution, shown by the dashed line, the mean radius for 
neutron production is $37.3 \mu \mathrm{m}$ while the radius within which $50 \%$ of the neutrons are produced is $28.1 \mu \mathrm{m}$. Looking at the fast component (solid curve) the mean radius for neutron production is $11.1 \mu \mathrm{m}$ while the radius within which $50 \%$ of the neutrons are produced is $9.8 \mu \mathrm{m}$.

\section{Conclusions}

Simulations done with Lasnex predict a mean ion temperature of $1.07 \mathrm{keV}$ in the center of a sphere of $\mathrm{CD}_{2}$ that is compressed to extremely high temperatures and densities in the Gbar series of NIF Discovery Science experiments. Preliminary comparisons with experiments show good agreement. The simulations also predict burn weighted mean densities of $33 \mathrm{~g} / \mathrm{cc}$, mean pressures of $25 \mathrm{Gbar}$, and mean radius of $37 \mu \mathrm{m}$. When the simulations are restricted to looking at the neutrons produced within 40 ps after the peak of neutron production the hot spot has even more extreme conditions, with mean pressure of $41 \mathrm{Gbar}$, mean temperature of $2.0 \mathrm{keV}$, mean density of $32 \mathrm{~g} / \mathrm{cc}$ at a mean radius of $11 \mu \mathrm{m}$. Calculations suggest that, with sufficient resolution and a better signal to noise ratio, two temperatures could be extracted from fitting the nTOF spectrum. The shape of the nTOF spectral wings could be used to measure the fast high temperature component while the main peak determines the slower lower temperature component. The neutron measurements are augmented by X-ray imaging experiments [14] that were also done to characterize the plasma.

Acknowledgements. This work was performed under the auspices of the U.S. Department of Energy by Lawrence Livermore National Laboratory under Contract DE-AC52-07NA27344 and supported by Laboratory Directed Research and Development Grant No. 13-ERD-073. The experiments on NIF were done under the NIF Discovery Science Program. We thank Bruce Remington for his advice during the preparation of this manuscript. 


\section{References}

[1] L. Stixrude, Phys. Rev. Lett. 108 (2012) 055505.

[2] S. Seager, et al., Astrophys. J. 669 (2007) 1279.

[3] V. E. Fortov, Extreme States of Matter on Earth and in the Cosmos, Springer-Verlag, Heidelberg, 2011, p. 224.

[4] A. L. Kritcher et al., High Energy Density Phys. 10 (2014) 27-34.

[5] G. B. Zimmerman and W. L. Kruer, Comments Plasma Phys. Controlled Fusion 2 (1975) 85.

[6] M. Rosen et al., High Energy Density Phys. 7 (2011) 180-190.

[7] Andrea Kritcher: private communications

[8] M. L. Adams and P. F. Nowak, J. Comput. Phys. 146 (1998) 366-403.

[9] S. F. Khan et al. "Measuring X-ray burn history with the Streaked Polar Instrumentation for Diagnosing Energetic Radiation (SPIDER) at the National Ignition Facility (NIF)", Proc. SPIE 8505, 850505 (2012).

[10] S. LePape et al., Phys. Rev. Lett. 112 (2014) 225002.

[11] M. Gatu Johnson et al., Rev. Sci. Instrum. 83 (2012) 10 D308.

[12] L. Ballabio, J. Källne, and G. Gorni, Nucl. Fusion 38 (1998) 1723-1735.

[13] J. Lindl et al., Phys. Plasmas 21 (2014) 020501.

[14] Benjamin Bachmann: private communications 


\section{Figure captions:}

Fig. 1. a) (Color online) Schematic of the experimental setup. Solid spherical target of $\mathrm{CD}_{2}$ is shock compressed using a hohlraum radiation drive, then backlit with $\sim 9 \mathrm{keV} \mathrm{Zn} \mathrm{He}-\alpha$ X-rays to generate a time-resolved ("streaked") 1D image of the shock compression vs time. b) Simulated radiograph for the $9 \mathrm{keV} \mathrm{X}$-ray backlighter versus time and radius showing the shock wave converging to the central hot-spot. The vertical axis covers $8 \mathrm{~ns}$ and the horizontal axis covers a diameter of $0.2-\mathrm{cm}$.

Fig. 2. Simulated radiation temperature versus time inside the radiation cavity (hohlraum).

Fig. 3. Simulated neutron production rate calculated for the $\mathrm{CD}_{2}$ spherical target versus time where time zero is the time of peak neutron production.

Fig. 4. (Color online) Snapshots of the simulated pressure versus radius of the $\mathrm{CD}_{2}$ spherical target every 20 psec from $\mathrm{t}=-20 \mathrm{ps}$ to $+100 \mathrm{ps}$. Time zero is the time of peak neutron production. The black short-dashed curve shows the incoming shock 20 psec before peak neutron production, the solid red curve is at peak neutron production, and the blue long-dashed curve shows the outgoing shock 40 psec after peak neutron production.

Fig. 5. (Color online) Snapshots of the calculated neutron production rate versus radius of the $\mathrm{CD}_{2}$ spherical target every 20 psec from $\mathrm{t}=-20 \mathrm{ps}$ to $+100 \mathrm{ps}$. Time zero is the time of peak neutron production. The black short-dashed curve is 20 psec before peak neutron production, the solid red curve is at peak neutron production, and the blue long-dashed curve is $40 \mathrm{psec}$ after peak neutron production.

Fig. 6. (Color online) Snapshots of the calculated ion temperature versus radius of the $\mathrm{CD}_{2}$ spherical target every 20 psec from $\mathrm{t}=-20 \mathrm{ps}$ to $+100 \mathrm{ps}$. Time zero is the time of peak neutron production. The black short-dashed curve is 20 psec before peak neutron production, the solid red curve is at peak neutron production, and the blue long-dashed curve is 40 psec after peak neutron production.

Fig. 7. (Color online) Snapshots of the calculated density versus radius of the $\mathrm{CD}_{2}$ spherical target every 20 psec from $\mathrm{t}=-20$ ps to +100 ps. Time zero is the time of peak neutron production. The black short-dashed curve is $20 \mathrm{psec}$ before peak neutron production, the solid red curve is at peak neutron production, and the blue long-dashed curve is 40 psec after peak neutron production.

Fig. 8. (Color online) Neutron time-of-flight (nTOF) spectrum for the nTOF diagnostic located 17.976 meters from the $\mathrm{CD}_{2}$ target. The experimental data is shown by the red solid curve while 
the simulation is shown by the black dashed curve: both are normalized to unity. The simulated results are broken into a fast and slow component that represents the neutrons created before and after +40 ps relative to the peak of neutron production.

Fig. 9. (Color online) Distribution function, dN/dP, for simulated production of $2.45 \mathrm{MeV}$ neutrons versus pressure $\mathrm{P}$. The black dashed curve shows the total distribution function over the entire calculation while the solid red curve (fast component) shows the results for a simulation that ends 40 psec after the peak of the neutron production.

Fig. 10. (Color online) Distribution function, $d N / d T_{i}$, for simulated production of $2.45 \mathrm{MeV}$ neutrons versus ion temperature $\mathrm{T}_{\mathrm{i}}$. The black dashed curve shows the total distribution function over the entire calculation while the solid red curve (fast component) shows the results for a simulation that ends $40 \mathrm{psec}$ after the peak of the neutron production. The high frequency oscillations above $\mathrm{T}_{\mathrm{i}} \sim 1.5 \mathrm{keV}$ are a numerical artifact.

Fig. 11. (Color online) Distribution function, $\mathrm{dN} / \mathrm{d} \rho$, for simulated production of $2.45 \mathrm{MeV}$ neutrons versus density $\rho$. The black dashed curve shows the total distribution function over the entire calculation while the solid red curve (fast component) shows the results for a simulation that ends 40 psec after the peak of the neutron production.

Fig. 12. (Color online) Distribution function, $\mathrm{dN} / \mathrm{dR}$, for simulated production of $2.45 \mathrm{MeV}$ neutrons versus radius $\mathrm{R}$. The black dashed curve shows the total distribution function over the entire calculation while the solid red curve (fast component) shows the results for a simulation that ends 40 psec after the peak of the neutron production. 


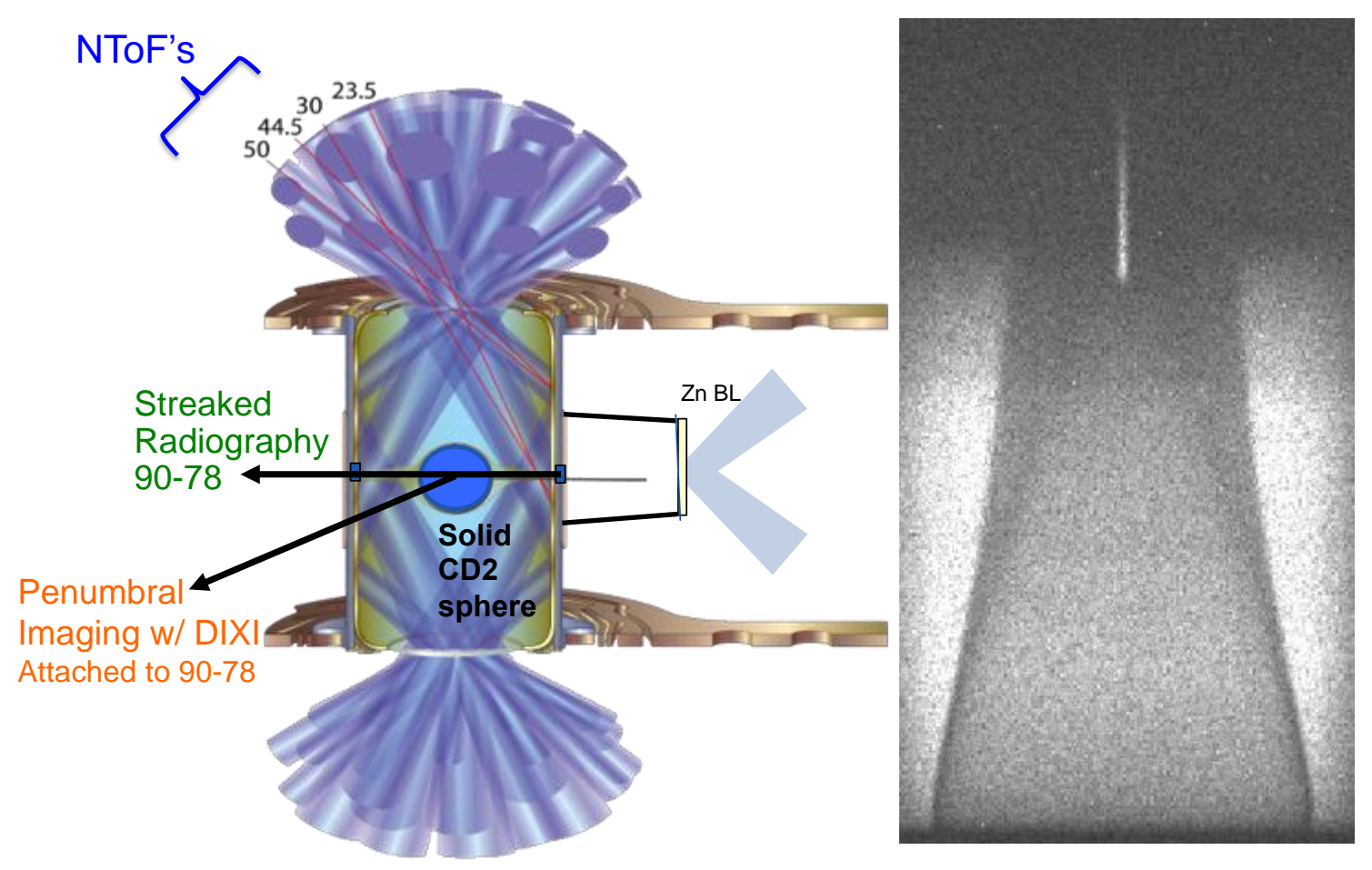

Fig. 1. a) (Color online) Schematic of the experimental setup. Solid spherical target of $\mathrm{CD}_{2}$ is shock compressed using a hohlraum radiation drive, then backlit with $\sim 9 \mathrm{keV} \mathrm{Zn} \mathrm{He}-\alpha \mathrm{X}$-rays to generate a time-resolved ("streaked") 1D image of the shock compression vs time. b) Simulated radiograph for the $9 \mathrm{keV} \mathrm{X-ray} \mathrm{backlighter} \mathrm{versus} \mathrm{time} \mathrm{and} \mathrm{radius} \mathrm{showing} \mathrm{the} \mathrm{shock} \mathrm{wave}$ converging to the central hot-spot. The vertical axis covers $8 \mathrm{~ns}$ and the horizontal axis covers a diameter of $0.2-\mathrm{cm}$. 


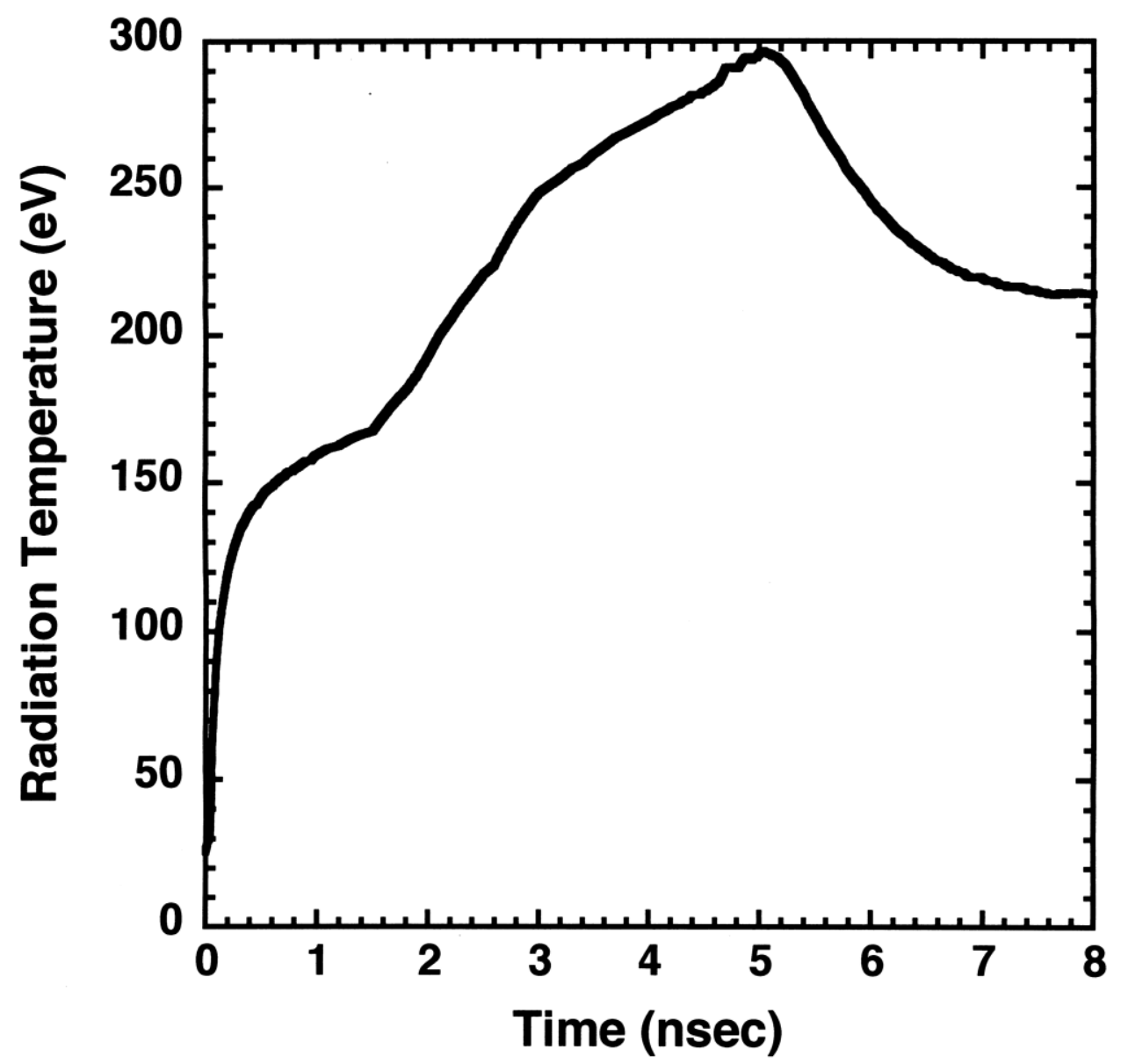

Fig. 2. Simulated radiation temperature versus time inside the radiation cavity (hohlraum). 


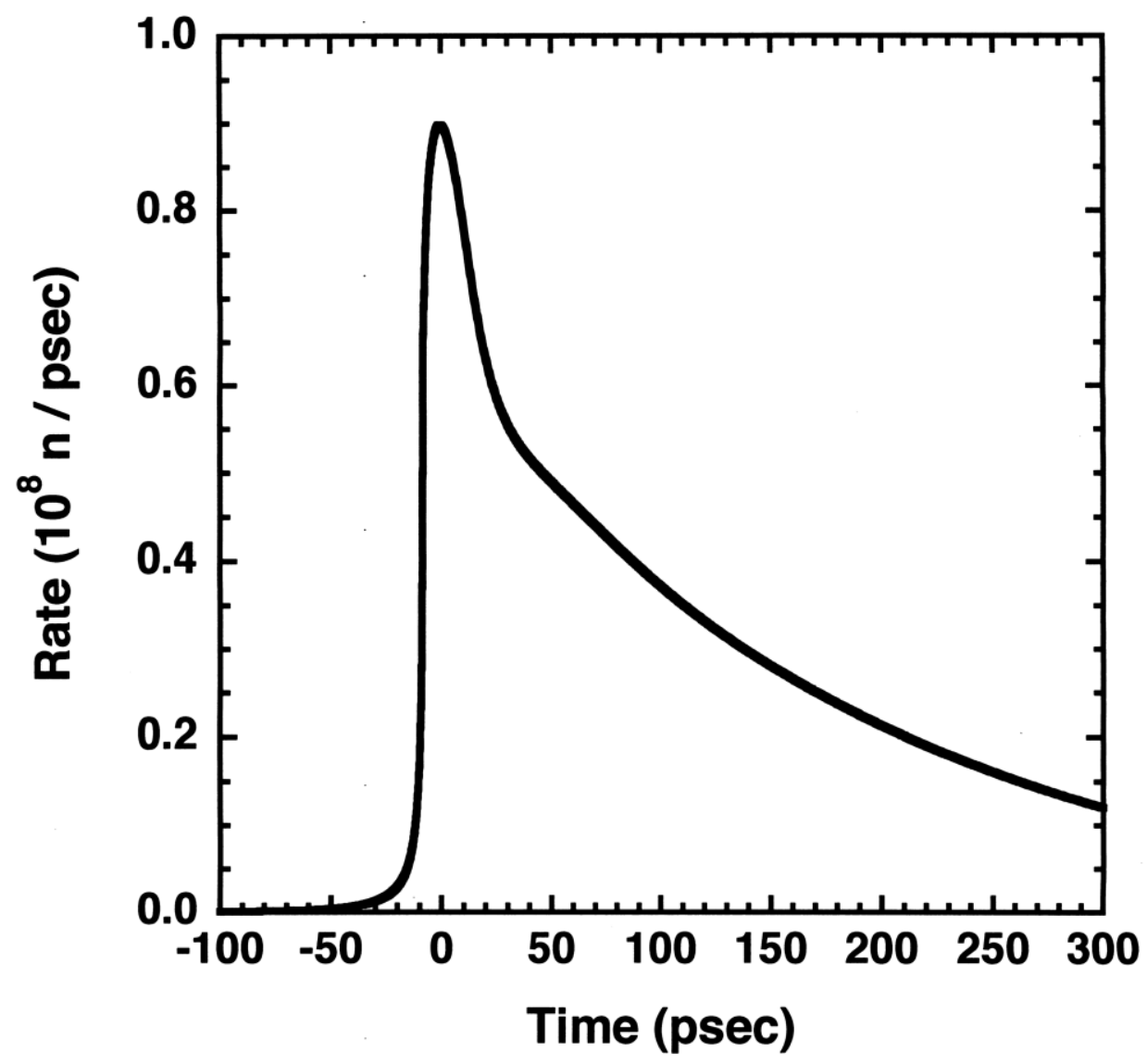

Fig. 3. Simulated neutron production rate calculated for the $\mathrm{CD}_{2}$ spherical target versus time where time zero is the time of peak neutron production. 


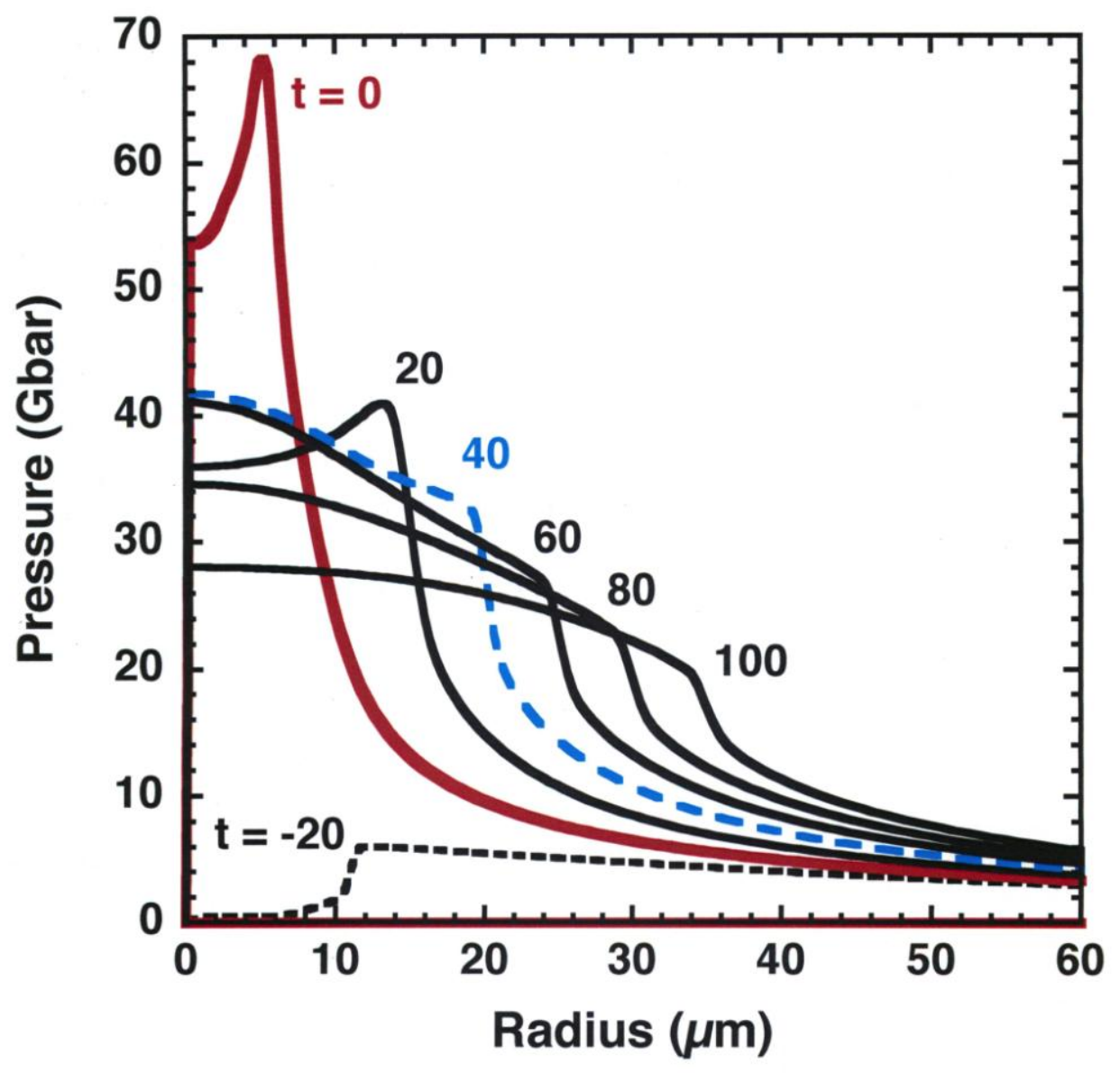

Fig. 4. (Color online) Snapshots of the simulated pressure versus radius of the $\mathrm{CD}_{2}$ spherical target every $20 \mathrm{psec}$ from $\mathrm{t}=-20 \mathrm{ps}$ to $+100 \mathrm{ps}$. Time zero is the time of peak neutron production. The black short-dashed curve shows the incoming shock 20 psec before peak neutron production, the solid red curve is at peak neutron production, and the blue long-dashed curve shows the outgoing shock 40 psec after peak neutron production. 


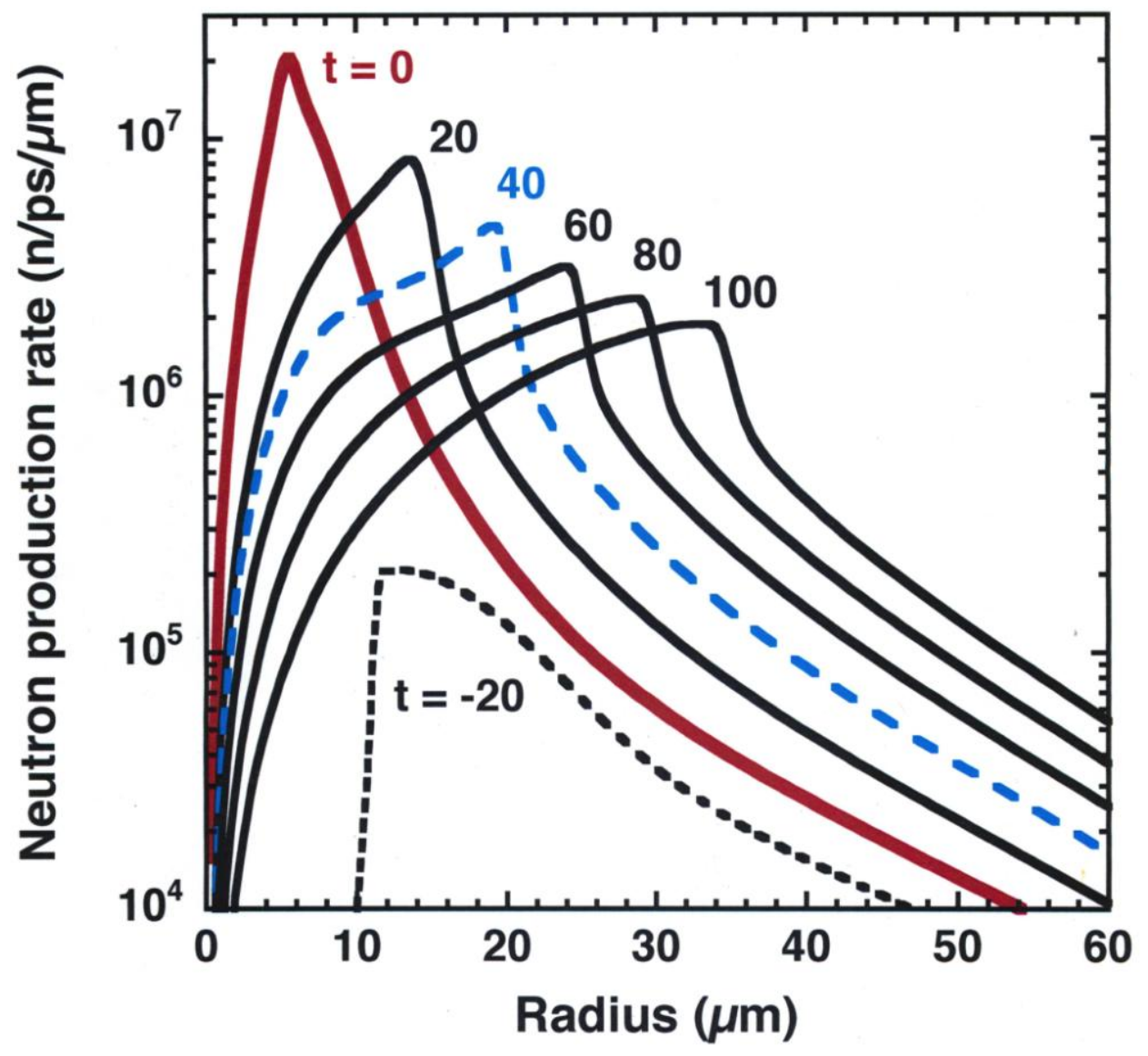

Fig. 5. (Color online) Snapshots of the calculated neutron production rate versus radius of the $\mathrm{CD}_{2}$ spherical target every $20 \mathrm{psec}$ from $\mathrm{t}=-20 \mathrm{ps}$ to $+100 \mathrm{ps}$. Time zero is the time of peak neutron production. The black short-dashed curve is $20 \mathrm{psec}$ before peak neutron production, the solid red curve is at peak neutron production, and the blue long-dashed curve is $40 \mathrm{psec}$ after peak neutron production. 


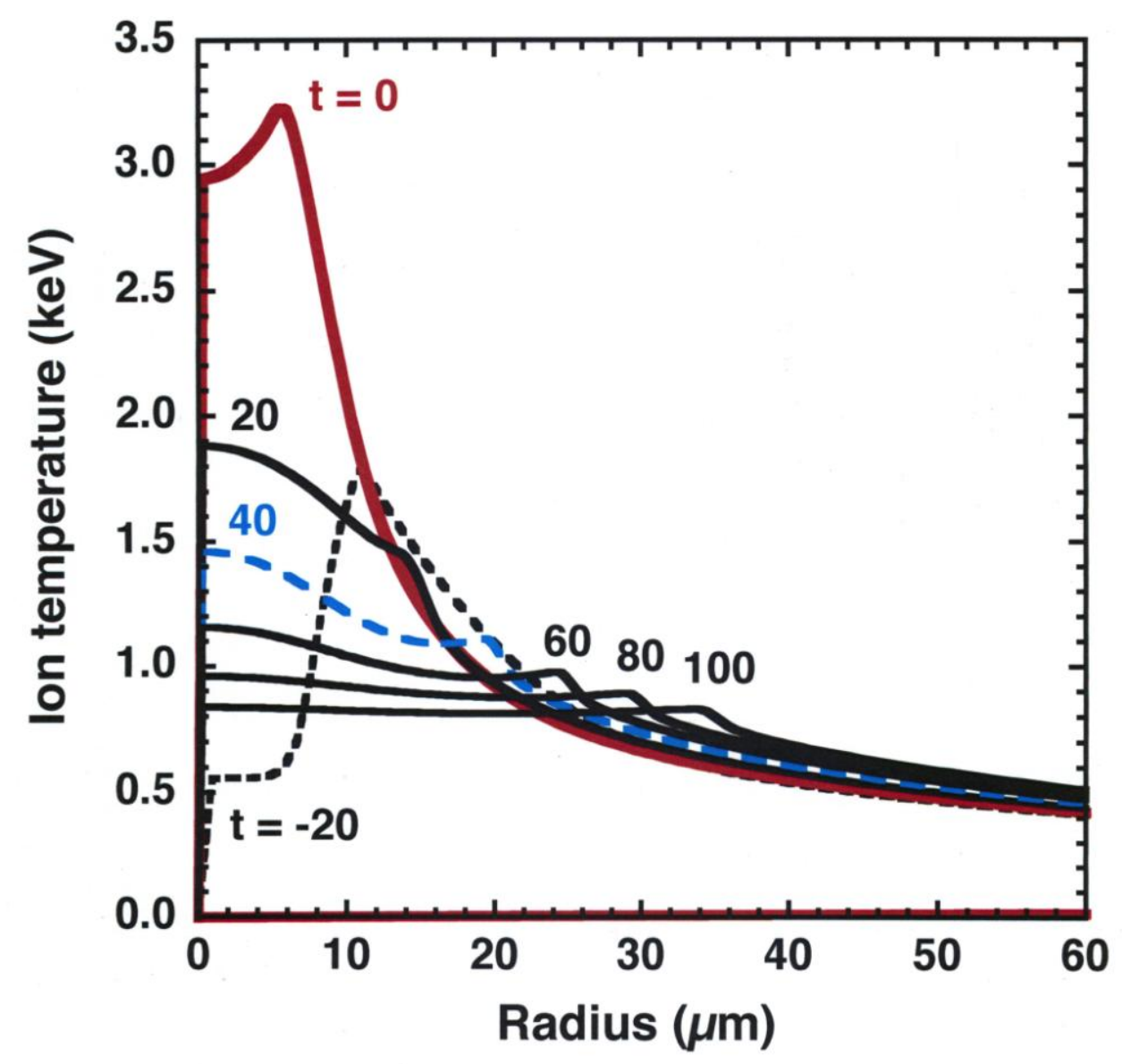

Fig. 6. (Color online) Snapshots of the calculated ion temperature versus radius of the $\mathrm{CD}_{2}$ spherical target every $20 \mathrm{psec}$ from $\mathrm{t}=-20 \mathrm{ps}$ to $+100 \mathrm{ps}$. Time zero is the time of peak neutron production. The black short-dashed curve is $20 \mathrm{psec}$ before peak neutron production, the solid red curve is at peak neutron production, and the blue long-dashed curve is $40 \mathrm{psec}$ after peak neutron production. 


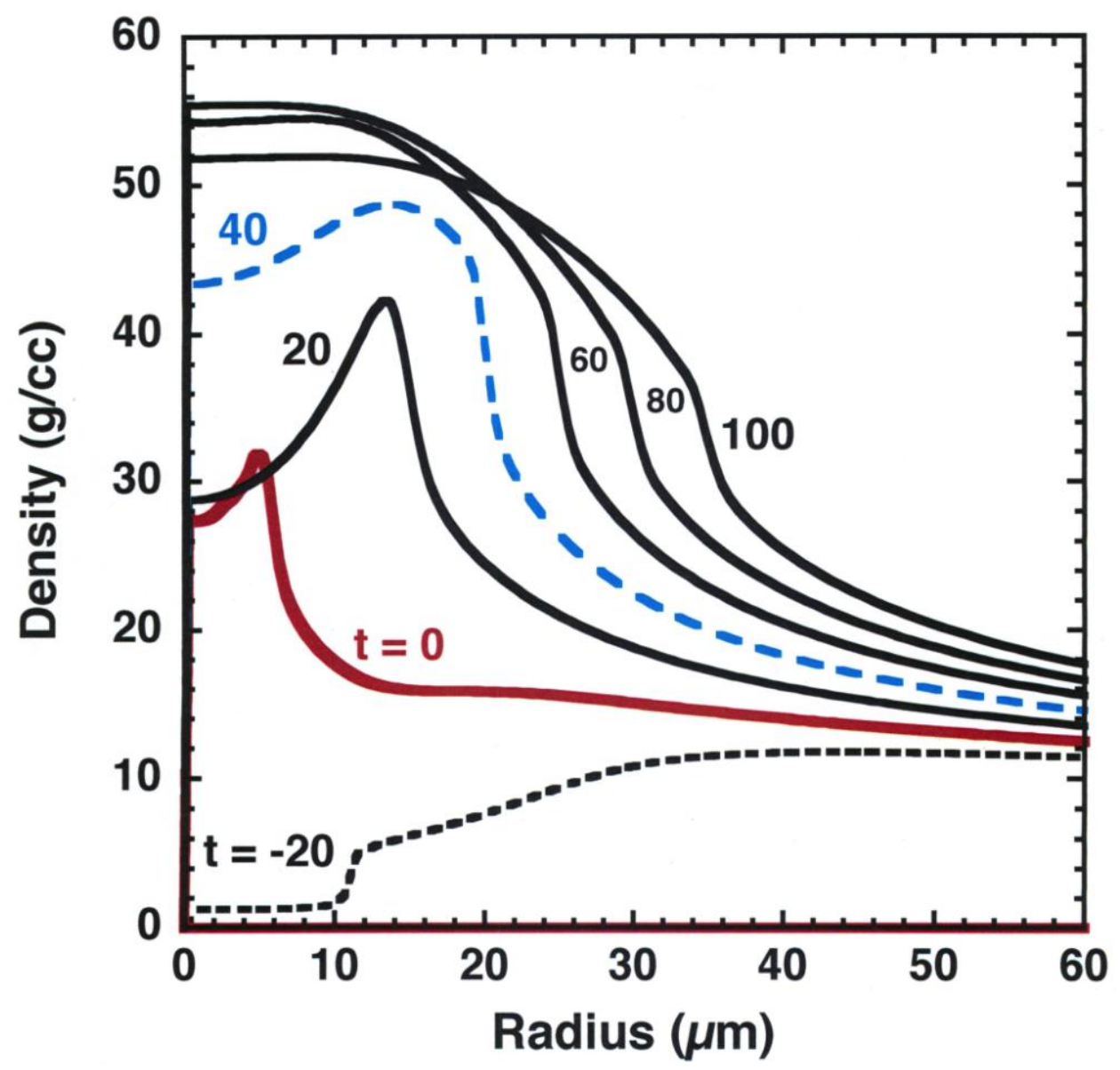

Fig. 7. (Color online) Snapshots of the calculated density versus radius of the $\mathrm{CD}_{2}$ spherical target every 20 psec from $\mathrm{t}=-20$ ps to $+100 \mathrm{ps}$. Time zero is the time of peak neutron production. The black short-dashed curve is 20 psec before peak neutron production, the solid red curve is at peak neutron production, and the blue long-dashed curve is 40 psec after peak neutron production. 


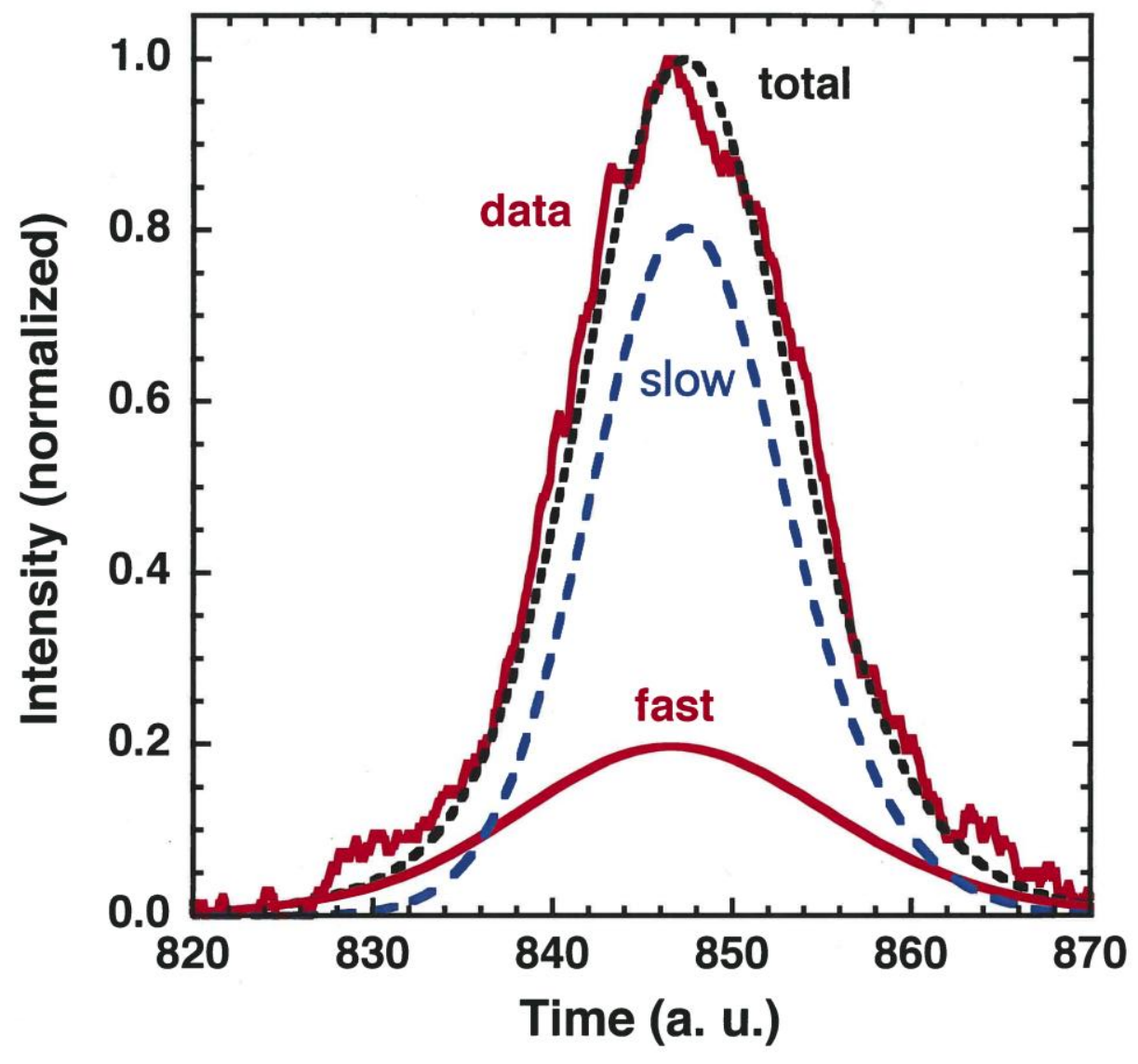

Fig. 8. (Color online) Neutron time-of-flight (nTOF) spectrum for the nTOF diagnostic located 17.976 meters from the $\mathrm{CD}_{2}$ target. The experimental data is shown by the red solid curve while the simulation is shown by the black dashed curve: both are normalized to unity. The simulated results are broken into a fast and slow component that represents the neutrons created before and after $+40 \mathrm{ps}$ relative to the peak of neutron production. 


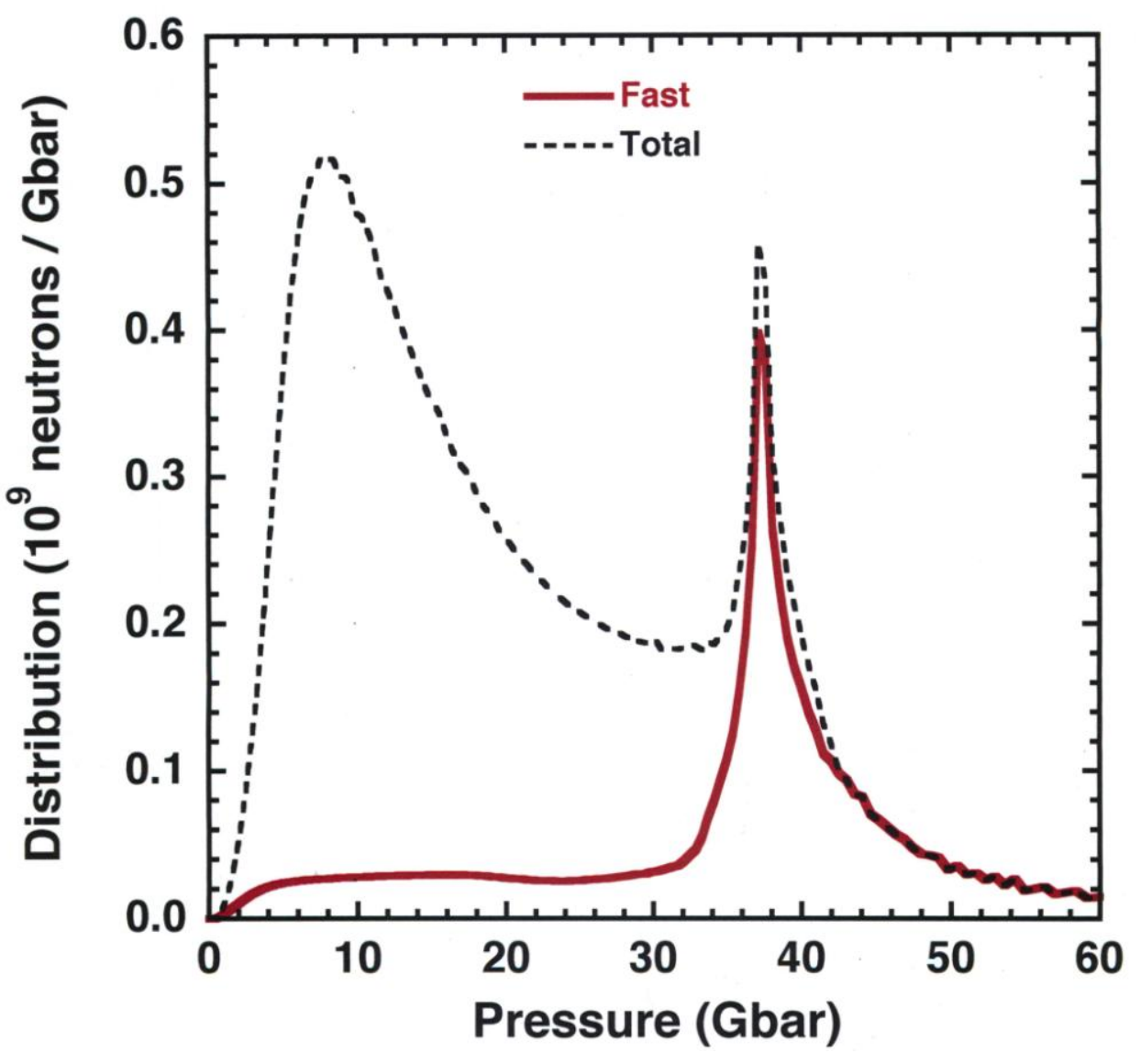

Fig. 9. (Color online) Distribution function, $\mathrm{dN} / \mathrm{dP}$, for simulated production of $2.45 \mathrm{MeV}$ neutrons versus pressure $P$. The black dashed curve shows the total distribution function over the entire calculation while the solid red curve (fast component) shows the results for a simulation that ends 40 psec after the peak of the neutron production. 


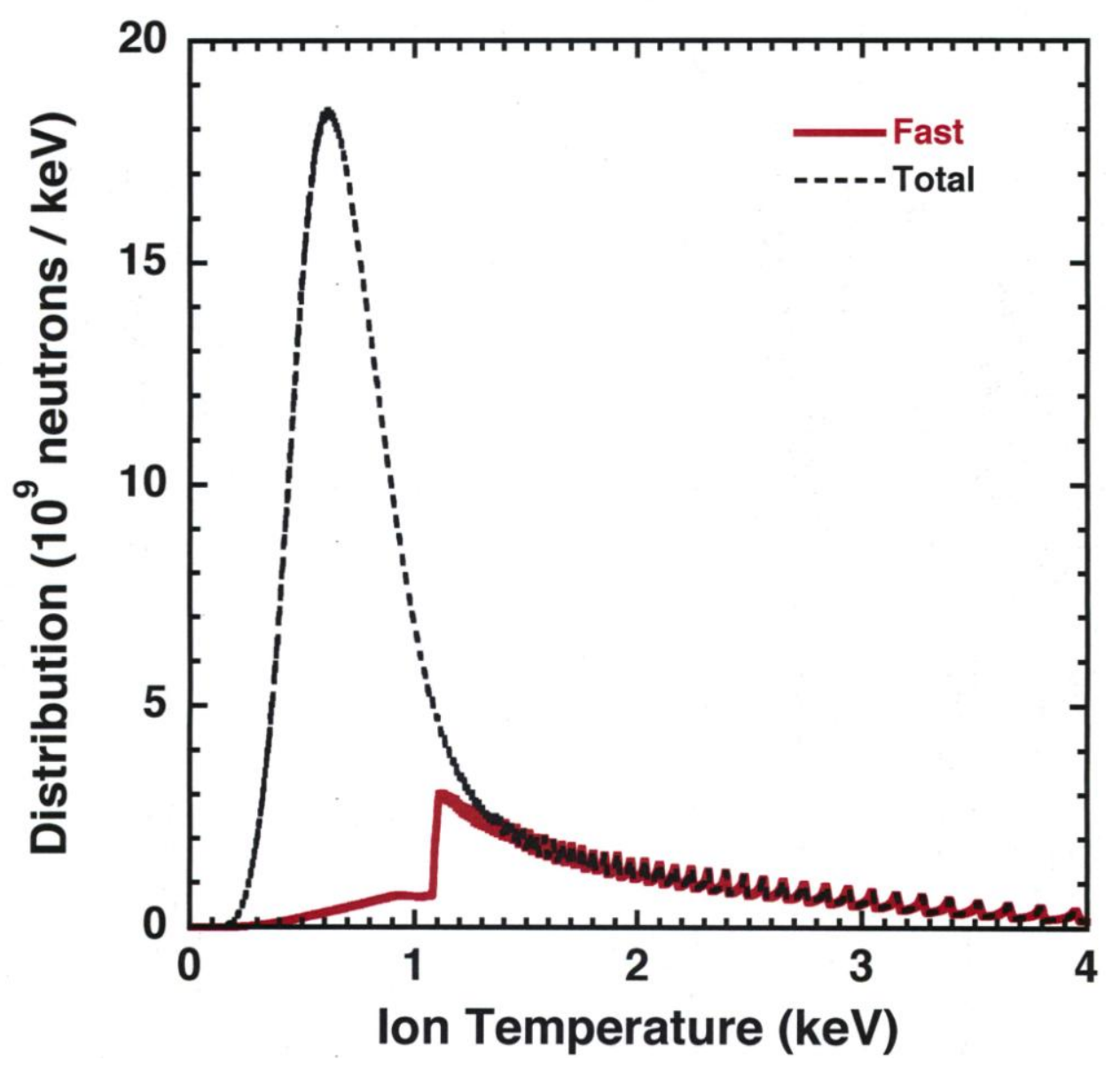

Fig. 10. (Color online) Distribution function, $\mathrm{dN} / \mathrm{dT}_{\mathrm{i}}$, for simulated production of $2.45 \mathrm{MeV}$ neutrons versus ion temperature $T_{i}$. The black dashed curve shows the total distribution function over the entire calculation while the solid red curve (fast component) shows the results for a simulation that ends $40 \mathrm{psec}$ after the peak of the neutron production. The high frequency oscillations above $\mathrm{T}_{\mathrm{i}} \sim 1.5 \mathrm{keV}$ are a numerical artifact. 


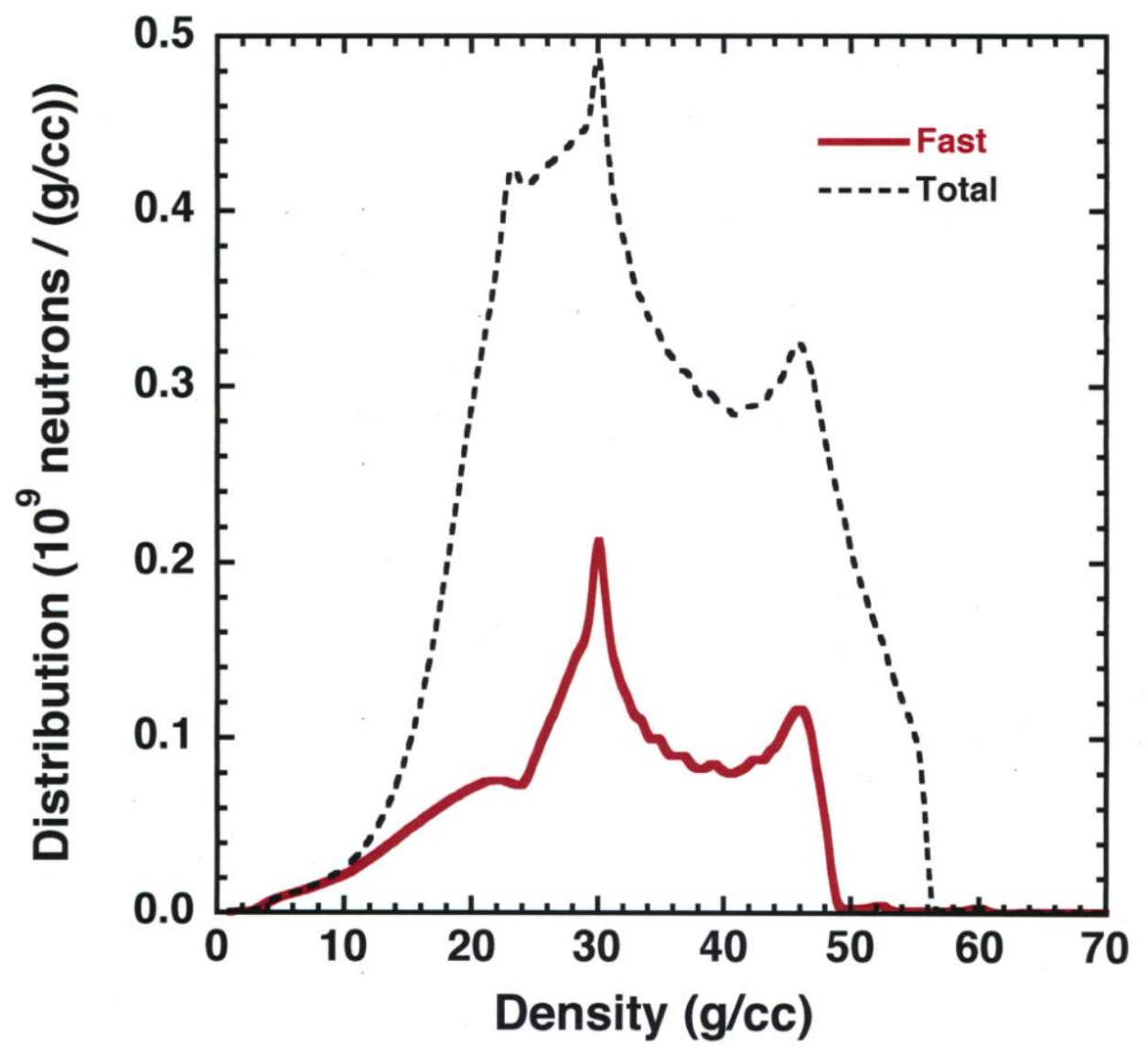

Fig. 11. (Color online) Distribution function, $\mathrm{dN} / \mathrm{d} \rho$, for simulated production of $2.45 \mathrm{MeV}$ neutrons versus density $\rho$. The black dashed curve shows the total distribution function over the entire calculation while the solid red curve (fast component) shows the results for a simulation that ends $40 \mathrm{psec}$ after the peak of the neutron production. 


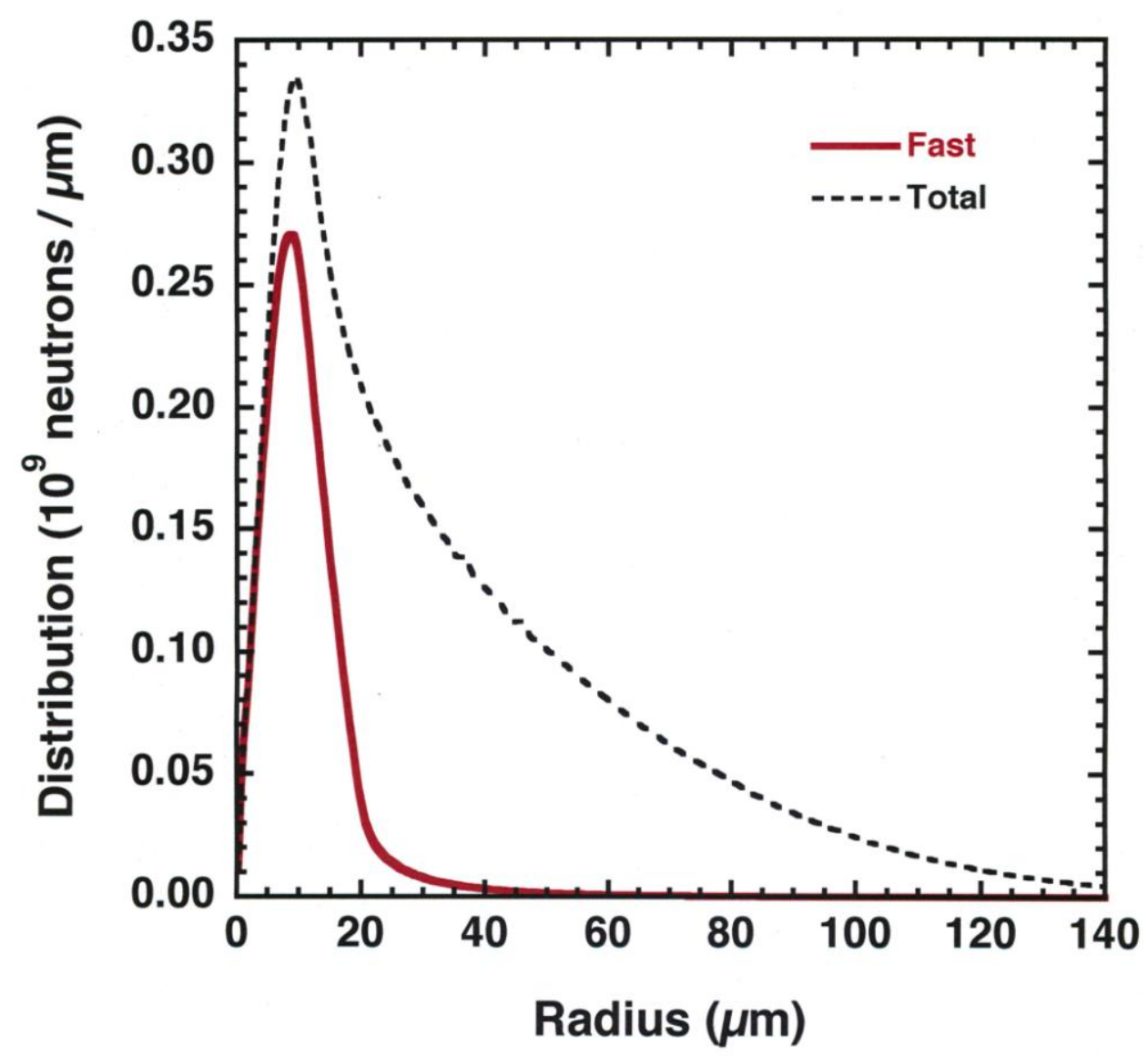

Fig. 12. (Color online) Distribution function, $d N / d R$, for simulated production of $2.45 \mathrm{MeV}$ neutrons versus radius $R$. The black dashed curve shows the total distribution function over the entire calculation while the solid red curve (fast component) shows the results for a simulation that ends $40 \mathrm{psec}$ after the peak of the neutron production. 